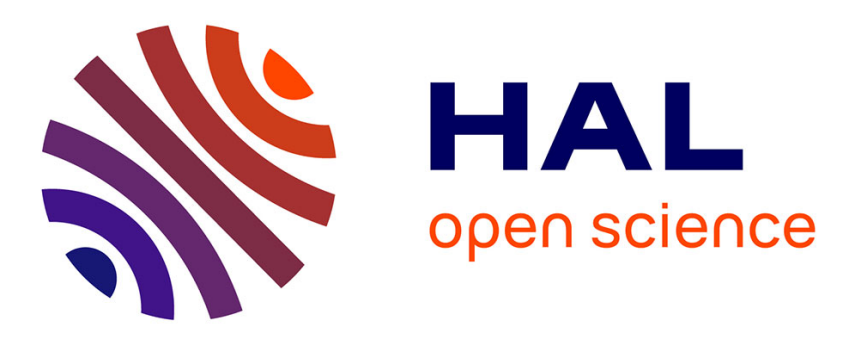

\title{
Système interactif de détection de comportements dynamiques anormaux
}

Benoit Rouxel, Franck Poirier, Gilles Coppin, Jean-Yves Antoine

\section{To cite this version:}

Benoit Rouxel, Franck Poirier, Gilles Coppin, Jean-Yves Antoine. Système interactif de détection de comportements dynamiques anormaux. Ergo'IHM 2012, Oct 2012, Biarritz, France. 6 pp. hal00788165

\section{HAL Id: hal-00788165 \\ https://hal.science/hal-00788165}

Submitted on 14 Feb 2013

HAL is a multi-disciplinary open access archive for the deposit and dissemination of scientific research documents, whether they are published or not. The documents may come from teaching and research institutions in France or abroad, or from public or private research centers.
L'archive ouverte pluridisciplinaire HAL, est destinée au dépôt et à la diffusion de documents scientifiques de niveau recherche, publiés ou non, émanant des établissements d'enseignement et de recherche français ou étrangers, des laboratoires publics ou privés. 


\section{Système interactif de détection de comportements dynamiques anormaux}

\author{
Benoit ROUXEL ${ }^{1}$ \\ benoit.rouxel@live.fr \\ Gilles COPPIN ${ }^{1}$ \\ gilles.coppin@telecom-bretagne.eu \\ ${ }^{(1)}$ Lab-STICC, TelecomBretagne \\ CS 83818, 29238 Brest

\section{RESUME} \\ Cet article présente une approche d'identification des \\ comportements anormaux dans le cadre de la surveillance \\ maritime. Après avoir rappelé les problèmes de surcharge \\ cognitive qui se posent aux opérateurs du contrôle \\ maritime, l'article présente le processus que les contrôleurs \\ mettent en œuvre pour analyser une situation. Il détaille \\ ensuite les approches d'identification existantes avant de \\ proposer une démarche originale qui inclut l'humain dans le \\ processus d'aide à la détection de situations anormales.
}

\author{
Franck POIRIER $^{2}$ \\ franck.poirier@univ-ubs.fr \\ Jean-Yves ANTOINE ${ }^{2,3}$ \\ jean-yves.antoine@univ-tours.fr
}

\author{
${ }^{(2)}$ Lab-STICC, UBS \\ BP 57356017 Vannes Cedex
}

\author{
${ }^{(3)}$ Université de Tours, LI \\ 3 place Jean Jaurès, 41000 Blois
}

\section{Mots-Clés}

Détection d'anomalies, interaction, système critique.

\section{ABSTRACT}

This paper presents an approach to identify abnormal behavior in a sea surveillance context. It presents the equipment used by sea surveillance operators. Then, it details the cognitive process followed by operators to analyze a situation as well as the identification techniques that have been investigated to support sea surveillance. At last, the paper propose an original approach that includes the human operator during the detection process.

\section{Author Keywords}

Anomaly detection, interaction, critical system,

\section{ACM Classification Keywords}

H.5.m. Information interfaces and presentation.

\section{General Terms}

Human Factors; Design.

\section{INTRODUCTION}

L'Union Européenne (UE) est une des zones les plus développées de la planète et l'une des zones incontournables du commerce mondial. Par conséquent, elle est aussi une des premières régions touchées par la

Permission to make digital or hard copies of all or part of this work for personal or classroom use is granted without fee provided that copies are not made or distributed for profit or commercial advantage and that copies bear this notice and the full citation on the first page. To copy otherwise, or republish, to post on servers or to redistribute to lists, requires prior specific permission and/or a fee.

Ergo'IHM 2012, October 16-19, 2012, Biarritz, France.

Copyright 2012 ACM 978-1-4503-1015-4/12/05...\$10.00. contrebande et potentiellement par les attaques terroristes. De par sa grande façade maritime, l'UE ne peut faire l'économie d'un système de surveillance de ses frontières maritimes.

Dans cet article, nous présenterons un système permettant aux opérateurs du contrôle maritime d'analyser les comportements des bateaux naviguant le long de la côte. Notre système accorde un rôle central aux contrôleurs dans le processus d'aide à la détection des trajectoires anormales. Il propose à ces derniers de définir au préalable, de façon intuitive et interactive, un ensemble de règles définissant les comportements suspects ou anormaux sur une zone donnée, de visualiser interactivement les trajectoires des bateaux et de détecter les anomalies, facilitant ainsi la surveillance d'une zone maritime.

Cet article est organisé comme suit: tout d'abord, nous présentons les équipements actuels à disposition des opérateurs pour mener à bien leurs missions ainsi que les principaux processus que ces derniers mettent en œuvre pour analyser une situation. Nous détaillons ensuite l'état de l'art en matière d'aide à la surveillance maritime. Nous introduisons ensuite en détail notre approche. Enfin, nous concluons et donnons quelques perspectives.

\section{CONTEXTE : CONTRÔLE MARITIME}

Les VTS (Vessel Traffic Service) sont les centres de contrôle à partir desquels le trafic maritime est surveillé. Ils ont pour but d'améliorer la sécurité et l'organisation du trafic ainsi que de protéger l'environnement.

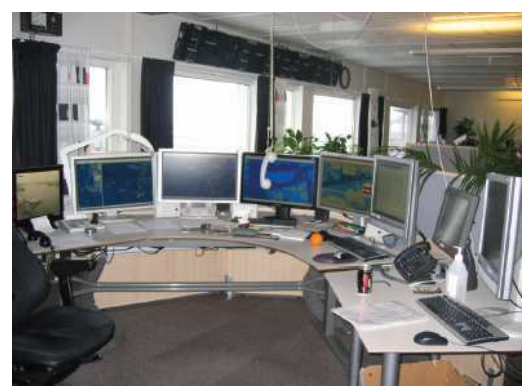

Figure 1. Poste de travail d'un opérateur dans un VTS. 
Les contrôleurs des VTS travaillent sur plusieurs moniteurs en même temps. La figure 1 nous présente le poste de travail d'un opérateur. Ce poste est composé de 4 moniteurs affichant respectivement des informations radar et AIS (le système d'identification automatique AIS permet entre autres d'identifier un bateau et de transmettre sa position GPS), des informations météorologiques, le planning portuaire et enfin la vidéo surveillance du (ou des) port(s) situé(s) dans la zone maritime surveillée. On remarque donc que l'opérateur doit traiter une masse considérable d'information brute.

Cette lourde charge cognitive réduit l'efficacité de l'opérateur. C'est pour réduire cette charge cognitive qu'ont été développés des systèmes d'aide à la prise de décision spécifiques au domaine de la surveillance maritime.

L'interview d'opérateurs [3] a permis de caractériser plusieurs règles génériques (portant sur la nature des navires et leur cinématique) de détection de situations anormales qui seraient susceptibles d'aider ces opérateurs dans leurs tâches. Il faut cependant être méfiant en ce qui concerne le choix des règles que l'on intègre au système. Ainsi, la mise en œuvre d'un système d'aide à la prévention des collisions, installé dans le VTS de Finisterre en Espagne, et intégrant ces règles, a rapidement montré [5] que ce dispositif déclenchait un trop grand nombre de fausses alertes. De plus, de par la nature même des missions des VTS, le travail des opérateurs est stressant. Ne comprenant pas bien les raisons du déclenchement des alertes du système et afin de ne pas ajouter une source de stress supplémentaire, le dispositif a finalement été désinstallé.

Il faut donc, avant même de proposer des solutions d'aide à la prise de décision pour les opérateurs, chercher à analyser le comportement des contrôleurs en situation écologique de surveillance pour proposer une aide.

\section{PROCESSUS D'ANALYSE DE SITUATIONS}

Comme l'expliquait un opérateur interrogé dans [5], la détection des comportements anormaux se déroule généralement selon un processus itératif et cyclique de raffinement de l'analyse de la situation, qui part d'une vision globale du trafic et qui descend jusqu'à l'identification précise des raisons d'un comportement anormal. On reconnaît dans cette approche les 3 principales étapes de ce que Schneiderman [6] appelle «le mantra de la visualisation interactive » qui s'énonce ainsi : «Partir d'une vue d'ensemble, puis zoomer et filtrer, et enfin d'accéder à l'information élémentaire à la demande ».

Pour Thomas et al. [7], le processus d'analyse d'une situation n'est pas un processus linéaire, mais bien cyclique et itératif, permettant de structurer progressivement l'information. On peut ainsi observer comment les données brutes fournies par les équipements de surveillance font sens et se transforment en connaissance de manière progressive. Cette dimension est rarement intégrée par les systèmes actuels d'aide à la surveillance maritime, qui se sont le plus souvent ancrés sur une analyse statistique directe des données brutes à disposition, et qui ne prennent que peu en compte l'expertise et les connaissances des opérateurs qui sont pourtant au cœur du traitement de la situation.

\section{APPROCHES EXISTANTES}

Il existe principalement deux approches différentes permettant d'aider les opérateurs à détecter des trajectoires anormales de bateaux.

\section{Caractérisation automatique des trajectoires}

Cette approche [1] est purement statistique. Son objectif est d'extraire, à partir de l'historique des trajectoires des bateaux ayant navigué sur une zone, une caractérisation statistique de ce qui peut/doit être considérée comme une trajectoire normale. Pour ce faire, on sélectionne des trajectoires similaires puis on calcule la trajectoire médiane de ce groupe de trajectoires ainsi qu'un couloir spatiotemporel. Le résultat est incorporé à un modèle définissant ce qu'est la normalité pour une trajectoire.

En contexte opérationnel, le système compare toute nouvelle trajectoire avec le modèle statistique. Si une trajectoire n'est pas comprise dans le couloir spatiotemporel ou bien si elle possède un indice de similarité avec la trajectoire médiane trop faible (les bateaux zigzaguant sur une voie de navigation auront une faible similitude avec la médiane calculée pour cette voie), le bateau est signalé à l'opérateur comme ayant une trajectoire anormale. Une limitation de l'approche est qu'elle ne permet pas au système de s'adapter à un nouveau contexte opérationnel tel que le changement des règles de navigation. Elle engendre de fait un nombre important de faux positifs qui peut entrainer le rejet du système par les opérateurs comme ce fut le cas pour le VTS de Finisterre [5].

\section{Caractérisation semi-automatique des trajectoires}

Cette seconde approche est basée sur l'apprentissage supervisé avec retour auprès des contrôleurs [2], [4]. Comme précédemment, elle établit un modèle statistique en utilisant l'historique des trajectoires sur une zone, mais elle utilise aussi la caractérisation établie par l'opérateur pour raffiner le modèle et aussi augmenter sa fiabilité.

Avant la mise en condition opérationnelle, le système commence par créer un modèle comportemental des bateaux en utilisant des trajectoires déjà caractérisées (trajectoires jugées normales ou anormales par un opérateur). En condition opérationnelle, chaque nouvelle trajectoire est confrontée au modèle et caractérisée automatiquement par le système. C'est ensuite à l'opérateur d'indiquer, après l'avoir analysée, si elle est normale ou non. Les indications apportées par ce dernier permettent de savoir s'il faut ou non raffiner le modèle. Comme indiqué en [2], on constate que les bateaux contrevenants modifient leurs comportements lors d'évolution technologique ou 
règlementaire afin de ne pas être repéré. L'opérateur se retrouve sans aide tant que le système ne s'est pas adapté au nouveau comportement. Le temps d'adaptation peut être assez long puisque l'apprentissage supervisé requiert la caractérisation par l'opérateur de multiples situations déviantes.

\section{AIDE CENTREE SUR L'UTILISATEUR}

Alors que les solutions précédentes s'articulent plutôt sur une démarche «ascendante », la particularité essentielle de notre approche est de prendre en compte les connaissances de l'opérateur en lui donnant les moyens de les exprimer sous forme de règles spatio-temporelles, au moyen d'une interaction directe avec la carte. Notre système d'aide de surveillance sera ainsi implanté sur une table tactile réunissant sur une seule interface l'ensemble des données actuellement dispersées sur plusieurs moniteurs. La vue intégrée qui en résultera devrait ainsi permettre une réduction de la charge perceptive des opérateurs.

L'approche proposée est mixte (c.-à-d. descendante et ascendante). Le fonctionnement descendant du système permet à l'opérateur de définir lui-même, par l'édition de nouvelles règles, les comportements qu'il ne souhaite pas voir dans une zone donnée. Tout bateau contrevenant à ces règles sera mis en évidence. La détection ascendante est ici limitée à l'identification de trajectoires présentant des anomalies évidentes, permettant de traiter de façon robuste les situations triviales. Par exemple si un bateau suit une voie de navigation tout en zigzaguant à l'intérieur de celleci, il doit être signalé à l'opérateur.

\section{Les règles}

L'édition des règles comportementales est réalisée par l'opérateur via une interaction avec la carte affichée sur la table tactile qui sert d'interface interactive de surveillance, à l'aide de gestes le plus souvent analogiques (voir Figure 2 ). Ces règles sont créées soit lors de la préparation de mission, soit dans un cadre opérationnel. Elles ont pour but de traduire les connaissances de l'opérateur dans le système.

Cinq types de règles seront proposés à l'opérateur dans un premier temps :

- Zone d'exclusion spatiale : Le bateau ne doit pas entrer dans la zone.

- Zone d'exclusion temporelle: Le bateau ne doit pas pouvoir entrer dans une zone avant un temps défini.

- Zone de non-stationnement : Le bateau ne doit pas rester dans la zone plus d'un certain temps.

- Virage brusque: Le bateau ne doit pas changer de cap trop rapidement (cf. Figure 2)

- Accélération soudaine: Le bateau ne doit pas changer trop rapidement de vitesse (cf. Figure 2)
On notera que l'usage de gestes analogiques permet d'envisager la composition de certaines de ces règles élémentaires.

Lors des interviews des contrôleurs maritimes ([3], [5]), on s'aperçoit que ces derniers cherchent à repérer des trajectoires anormales. C'est pourquoi les règles n'ont pas pour but de définir un comportement normal, mais au contraire d'établir les caractéristiques d'une trajectoire anormale. Chaque règle créée est rattachée à un ou plusieurs types de bateau, à une zone et à un opérateur. Chaque opérateur peut ainsi définir les règles de détection qui lui semblent les plus pertinentes, répondant ainsi au fait que chaque opérateur analyse différemment une situation [3].

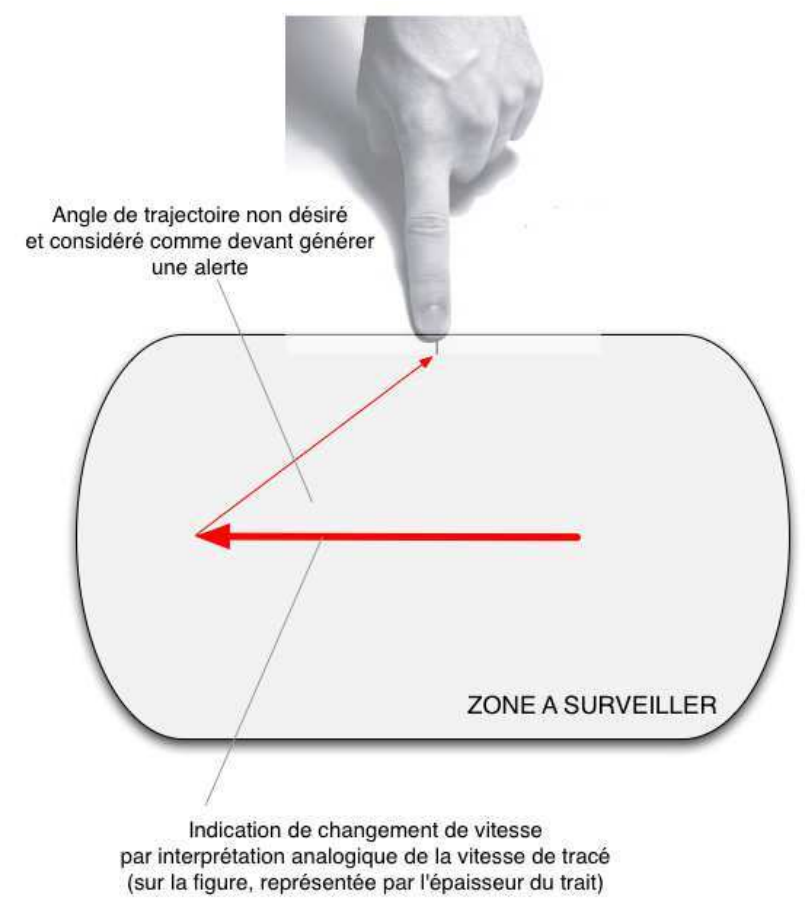

Figure 2 : exemple de définition de règle par geste analogique

\section{Le fonctionnement}

Comme le montre la figure 2, lorsque le contrôleur démarre le système, il a le choix entre passer en mode d'édition de règles (préparation de mission) ou en mode opérationnel.

En préparation de mission, l'opérateur commence donc par créer des règles. Le système confronte ces nouvelles règles à un historique de trajectoire caractérisées afin d'estimer leur pertinence. Cette évaluation permet à l'opérateur d'avoir une idée du nombre de faux positifs et de faux négatifs qu'engendrera la nouvelle règle. Il peut ainsi décider de modifier les paramètres de la règle pour accroitre ses performances. Si l'on prend par exemple une règle de type zone d'exclusion temporelle, l'opérateur peut décider d'augmenter le temps en dessous duquel le bateau ne doit pas pouvoir entrer dans la zone pour ne laisser passer 
aucune menace ou au contraire diminuer ce temps pour diminuer de nombre de fausses alertes. Une fois satisfait par l'évaluation de la règle, il peut la valider. Le système peut alors être basculé en mode opérationnel.

En mode opérationnel, si une trajectoire de bateau viole une des règles définies par l'opérateur ou présente un comportement anormal évident (c.-à-d. enfreint le modèle statistique purement ascendant), le bateau est mis en évidence sur l'interface. Le reste du temps, les bateaux sont affichés normalement sur la carte.

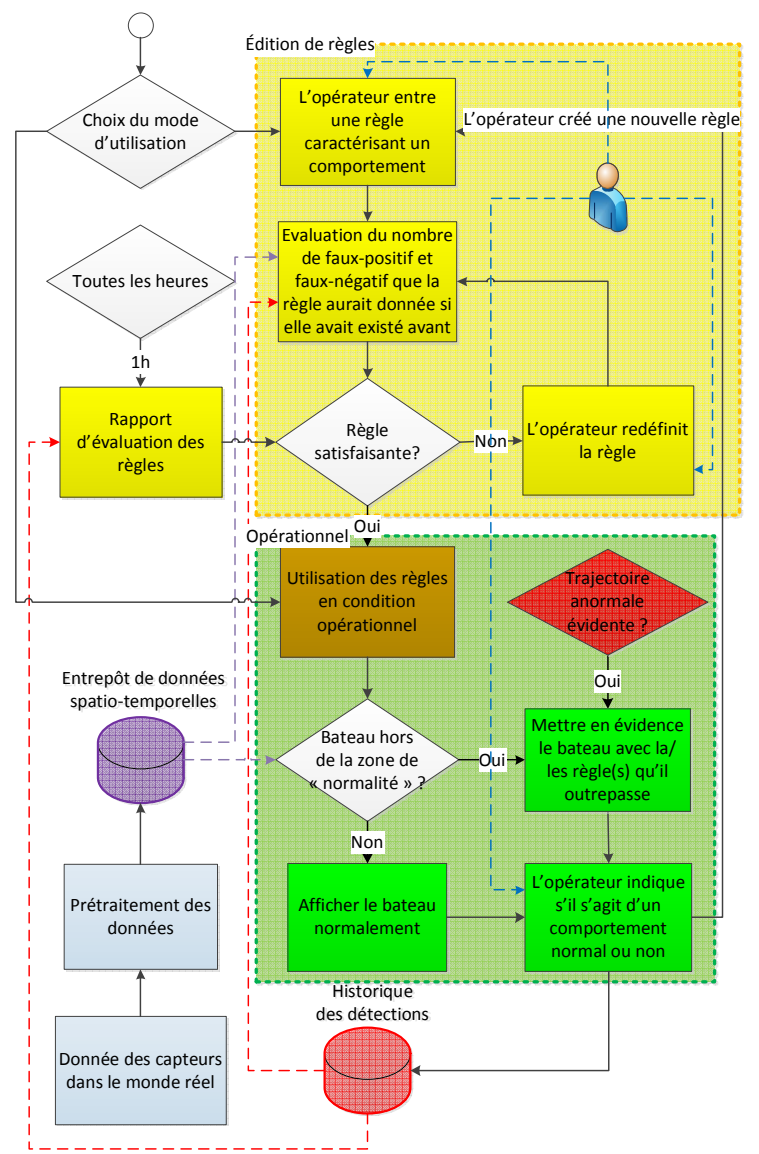

Figure 3. Fonctionnement et édition des règles dans le système

$\mathrm{Si}$ un bateau n'est pas reconnu comme ayant un comportement anormal, mais qu'après analyse, l'opérateur en déduit qu'il s'agit d'une trajectoire anormale, ce dernier peut ajouter une nouvelle règle rattachée à son compte afin de détecter ce nouveau type d'anomalie ultérieurement. Pour ce faire, on affiche les éléments caractéristiques de la trajectoire (changement de cap, de vitesse...) afin que l'opérateur puisse indiquer au système quels sont les éléments le gênant. Lorsque tous les éléments ont été sélectionnés, l'opérateur valide son choix et une règle propre à la zone et à l'opérateur est mise en œuvre. Une fois la mission terminée, les opérateurs peuvent se réunir et évaluer les règles créées par partir d'une trajectoire anormale. Lorsqu'ils jugent la nouvelle règle pertinente, ils peuvent créer un nouveau type de règle qui pourra être utilisé par tout le monde. L'avantage de cette approche est qu'elle devrait permettre aux opérateurs des VTS de décider eux même des types de comportement anormaux qu'ils souhaitent laisser à la charge de la machine. Compte tenu du fait que la création et l'évaluation de la règle sont à la charge de l'opérateur, on peut espérer que les alertes soient plus pertinentes au sens où elles correspondront exactement à son analyse idiosyncrasique de la situation. L'autre avantage de cette approche est qu'elle devrait permettre une appropriation plus rapide du système en ce qui concerne la détection de nouveaux types de comportement anormaux.

\section{CONCLUSION ET PERSPECTIVES}

Dans cet article, nous avons vu quels sont les équipements à disposition des contrôleurs maritime au sein des VTS à l'heure actuelle. Nous avons ensuite présenté un bref état de l'art sur nos connaissances actuelles sur le processus d'un analyste pour analyser une situation et sur les approches existantes afin de les aider dans la réalisation de leurs missions. Nous avons enfin présenté une approche qui utilise les connaissances de l'opérateur en l'incluant dans le processus d'identification des trajectoires anormales.

L'approche proposée doit maintenant être implémentée et testée afin qu'elle soit évaluée. Pour cela, nous allons réaliser un prototype sur table interactive permettant aux opérateurs d'éditer des règles appartenant à l'un des cinq types de règles énoncées dans ce papier. Par la suite, un effort sur la visualisation des informations devra être fourni afin de permettre à l'opérateur de comprendre rapidement et facilement les informations qui lui seront transmises.

\section{REFERENCES}

1. Etienne, L. Motifs spatio-temporels de trajectoires d'objets mobiles, de l'extraction à la détection de comportements inhabituels. Recherche, 2011, 190.

2. Morel, M., Napoli, A., George, J., and Jangal, F. Surveillance et contrôle des activités des navires en mer ScanMaris. Sensors (Peterborough, NH), (2010), $1-10$.

3. Nilsson, M., Laere, J.V., and Ziemke, T. Extracting rules from expert operators to support situation awareness in maritime surveillance. Fusion, 2008 11th, (2008), 908-915.

4. Riveiro, M., Falkman, G., and Ziemke, T. Improving maritime anomaly detection and situation awareness through interactive visualization. Information Fusion, 2008 11th International Conference on, IEEE, 1-8.

5. Riveiro, M. Visual Analytics for Maritime Anomaly Detection. Technology, 2011, 208.

6. Shneiderman, B. The eyes have it: A task by data type taxonomy for information visualizations. Visual Languages, 1996. Proceedings., IEEE, (1996).

7. Thomas, J.J. and Cook, K.A. Illuminating the path: The research and development agenda for visual analytics. IEEE, 2005. 
